\title{
Replicator Dynamics in Protocells
}

\author{
Peter F. Stadler ${ }^{a, b, c}$ and Bärbel M. R. Stadler ${ }^{d}$ \\ a Bioinformatics Group, Department of Computer Science, and \\ Interdisciplinary Center for Bioinformatics, University of Leipzig, \\ Härtelstraße 16-18, D-04107 Leipzig, Germany \\ studla@bioinf.uni-leipzig.de \\ ${ }^{\mathrm{b}}$ Department of Theoretical Chemistry and Structural Biology, University of \\ Vienna, \\ Währingerstraße 17, A-1090 Wien, Austria \\ ${ }^{\mathrm{c}}$ The Santa Fe Institute, 1399 Hyde Park Rd., Santa Fe NM 87501 \\ ${ }^{\mathrm{d}}$ Max Planck Institute for Mathematics in the Sciences \\ Inselstrasse 22, D-04103, Leipzig, Germany \\ stadler@mis.mpg.de
}

\begin{abstract}
Replicator equations have been studied for three decades as a generic dynamical system modelling replication processes. Here we show how they arise naturally in models of self-replicating polymers and discuss some of their basic properties. We then concentrate on a minimal dynamic model of a protocell by coupling replicating polymers with a growing membrane.
\end{abstract}

\section{Introduction}

In recent years, substantial progress has been made in understanding the requirements for minimal cell-like structures. Several proposals for artificial minimal cells have been put forward (Luisi et al., 1994; Pohorille \& Deamer, 2002; Szostak et al., 2001; Rasmussen et al., 2003). Some of them call for a sophisticated molecular machinery to be enclosed in a lipid vesicle. The model of Szostak et al. (Szostak et al., 2001) consists of a vesicle containing an RNA genome that contains an RNA-replicase ribozyme (e.g. an advanced version of the molecule described in (Johnston et al., 2001; Paul \& Joyce, 2003; Lawrence \& Bartel, 2005)) and a functionality that influences the fitness of the vesicle. The construct of Pohorille \& Deamer (Pohorille \& Deamer, 2002), which is even closer to a modern cell, includes transcription and translation functionalities. In contrast, the "LANL Bug" (Rasmussen et al., 2003) envisions a very simple genetic material in lipid aggregates that actively facilitates 
an autocatalytic reproduction of lipids as well as the genetic material itself. It is designed as a minimalistic, thermodynamic coupling between the three functional structures container, metabolism, and genes.

The integration of these fundamental building blocks requires a detailed knowledge of dynamic properties of each of the subsystems and their interactions. While advances in numerical mathematics make it feasible to simulate such systems, a structural analysis of the kinetic equations is a necessary prerequisite for understanding the principles upon which life-like physico-chemical structures operate.

Mathematically, the best-studied subsystem is autocatalytic replication. Template-dependent replication at the molecular level is the basis of reproduction in nature. Indeed, a plausible way of characterizing the origin of life is the emergence of heritable information that, through the interplay of selection and variation, leads to Darwinian evolution (Joyce, 2002). A detailed understanding of the peculiarities of the chemical reaction kinetics associated with replication processes is therefore an indispensable prerequisite for any understanding of evolution at the molecular level.

The notion of a replicator - originally invented by Richard Dawkins (Dawkins, 1976, pp.13-21) — is now used in biology for "an entity that passes on its structure largely intact in successive replications" (Vrba, 1989). Before we turn to the mathematics of replication processes, however, we very briefly summarize some of the experimental evidence for replication at the molecular level.

\section{Molecular Replicators}

Enzyme Catalyzed Replication of nucleic acids is today an ubiquitous technique in molecular biology. The most prominent example is the polymerase chain reaction (PCR). However, the first successful attempts to study RNA evolution in vitro were already carried out in the late sixties (Mills et al., 1967; Spiegelman, 1971) using the replicase enzyme of the bacteriophage $\mathrm{Q} \beta$. Extensive studies on the reaction kinetics of RNA replication in the $\mathrm{Q} \beta$ system revealed kinetic data consistent with a multi-step reaction mechanism (Biebricher et al., 1983; Biebricher \& Eigen, 1988). Depending on the concentration of template molecules, [C], one can distinguish three phases of the replication process: (i) at low concentration all free template molecules are instantaneously bound by the replicase, $\mathrm{E}$, which is present in excess and therefore the template concentration grows exponentially, (ii) excess of template molecules leads to saturation of enzyme molecules, then the rate of RNA synthesis becomes constant and the concentration of the template grows linearly, and (iii) very high template concentrations impede dissociation of the 
complexes between template and replicase, and the template concentration approaches a constant. This effect is known as product inhibition. We neglect plus-minus complementarity in replication by assuming stationarity in relative concentrations of plus and minus strand (Eigen, 1971) and consider the plusminus ensemble as a single species. Then, RNA replication in the $\mathrm{Q} \beta$ system may be described by the over-all mechanism:

$$
\mathrm{A}+\mathrm{C}+\mathrm{E} \underset{\bar{k}}{\stackrel{k}{\rightleftharpoons}} \mathrm{A}+\mathrm{C} \cdot \mathrm{E} \stackrel{a}{\longrightarrow} \mathrm{C} \cdot \mathrm{E} \cdot \mathrm{C} \underset{\bar{k}^{\prime}}{\stackrel{k^{\prime}}{\rightleftharpoons}} \mathrm{C} \cdot \mathrm{E}+\mathrm{C}
$$

Here A represents the building blocks, the dot indicates a non-covalently bond complex, and as before, $\mathrm{C}$ and $\mathrm{E}$, are template and replicase, respectively. Lowercase letter above or below the reaction arrows represent the reaction rate constants. This simplified reaction scheme reproduces all three characteristic phases of the detailed mechanism and can be readily extended to replication and mutation.

Minimal Molecular Replicators typically consist of a template and two substrate molecules that become joined to form a copy of the template. A number of experimental examples of such systems have been described so far, based on nucleic acids (von Kiedrowski, 1986; Zielinski \& Orgel, 1987; Paul \& Joyce, 2003), peptides (Lee et al., 1996, 1997; Yao et al., 1998; Isaac \& Chmieleswski, 2002; Ashkenazy et al., 2004), and small organic molecules (Tijvikua et al., 1990; Wintner et al., 1994), see (Paul \& Joyce, 2004) for a recent review.

The ligation-based mechanism of all these experimental systems is encapsulated by a common chemical reaction scheme. Here $C$ is the template, $A$ and $B$ are the building blocks, and $A B C$ denotes the complex in which $A$ and $B$ are properly aligned to the template $C$. The irreversible step is the ligation reaction, which converts $A B C$ into $C_{2}$. The complete system of chemical reactions reads

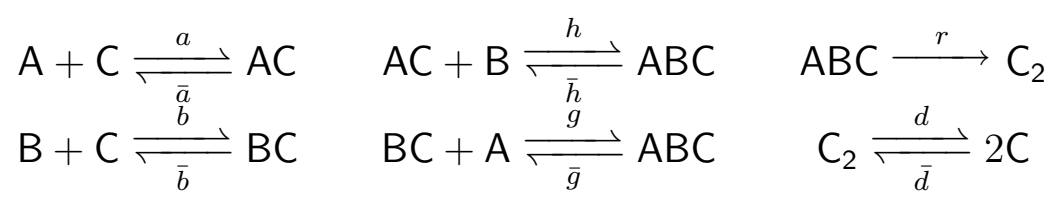

Note the difference between $2 \mathrm{C}$ (two isolated copies of the molecule $\mathrm{C}$ ) and $\mathrm{C}_{2}$ (the complex formed from two hybridized copies of $\mathrm{C}$ ).

A quite different mechanism of replication proceeds via DNA triple helices (Li \& Nicolaou, 1994): A DNA duplex C.C is replicated by first forming an adduct $C \cdot C^{\prime} D E$ with triple helix geometry, where the template strand forms standard Watson-Crick pairs, while the building blocks D and E are attached via Hoogsteen pairs. The fragments are ligated and then the resulting C. $C^{\prime} \mathrm{C}$ complex dissociates along the weaker Hoogsteen pairs. Finally, the single stranded template sequence is ligated with fragments of its complements 


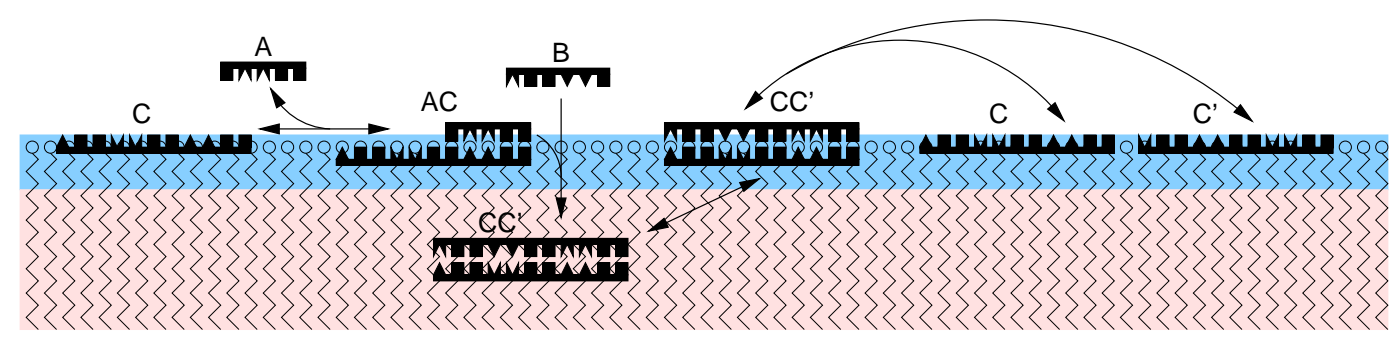

Fig. 1. Ligase-based replication reaction anchored in a lipid aggregate corresponding to eqn.(4). Adapted from (Rasmussen et al., 2004).

and forms a copy of the original duplex DNA. The reaction mechanism can be summarized as follows

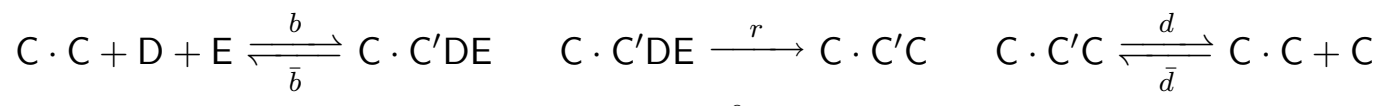

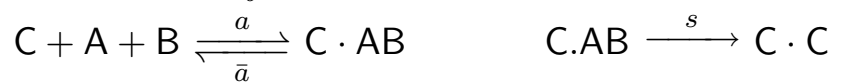

The proposed LANL Bug (Rasmussen et al., 2003) envisions simpler molecules, such as peptide nucleic acids (PNA) (Nielsen, 1993) as the genetic material. PNAs should be much easier to couple with a lipid layer than traditional nucleic acids due to their hydrophobic backbone. Note, however, that the standard PNA backbone will need to be modified using hydrophobic amino acids for this purpose. As in the other protocell proposals, it utilizes the lipid to keep the cooperative structure together. In contrast to other proposals, the proto-genes directly interact with the lipid; this requires a less sophisticated spatial organisation than vesicles (see e.g. (Apel et al., 2002)), making micelles (Whitten et al., 1998) or even less organized lipid aggregates plausible. A scheme of the replication mechanism is shown in Fig. 1. The overall reaction mechanism for this model can be summarized as follows:

$$
\begin{array}{lll}
\mathrm{A}+\mathrm{C} \underset{\bar{k}_{A}}{\stackrel{k_{A}}{\rightleftharpoons}} \mathrm{AC} & \mathrm{AC}+\mathrm{B} \stackrel{a^{\prime}}{\longrightarrow} \mathrm{C}_{2}{ }^{*} & \mathrm{C}_{2}{ }^{*} \stackrel{f}{\underset{\bar{f}}{\rightleftharpoons}} \mathrm{C}_{2} \\
\mathrm{~B}+\mathrm{C} \underset{\bar{k}_{B}}{\stackrel{k_{B}}{\rightleftharpoons}} \mathrm{BC} & \mathrm{BC}+\mathrm{A} \stackrel{a^{\prime \prime}}{\longrightarrow} \mathrm{C}_{2}{ }^{*} & \mathrm{C}_{2} \frac{k_{d}}{\underset{\bar{k}_{d}}{\rightleftharpoons}} 2 \mathrm{C}
\end{array}
$$

Here $C_{2}{ }^{*}$ denotes the duplex buried in the lipid phase, while $C_{2}$ denotes the duplex exposed on the surface where dissociation is thermodynamically feasible. The mechanism envisaged here is only one of several possibilities. Alternatively, one could assume that the $C^{\prime} C^{\prime}$ duplex dissociates already in the hydrophobic phase. In this case we have to consider the phase equilibrium of the template molecules rather than of the duplexes:

$$
\mathrm{C}_{2} * \frac{k_{d}^{*}}{\stackrel{\bar{k}_{d}^{*}}{\rightleftharpoons}} 2 \mathrm{C}^{*} \quad \text { and } \quad \mathrm{C}^{*} \frac{f^{\prime}}{\underset{\bar{f}^{\prime}}{\rightleftharpoons}} \mathrm{C} \text {. }
$$




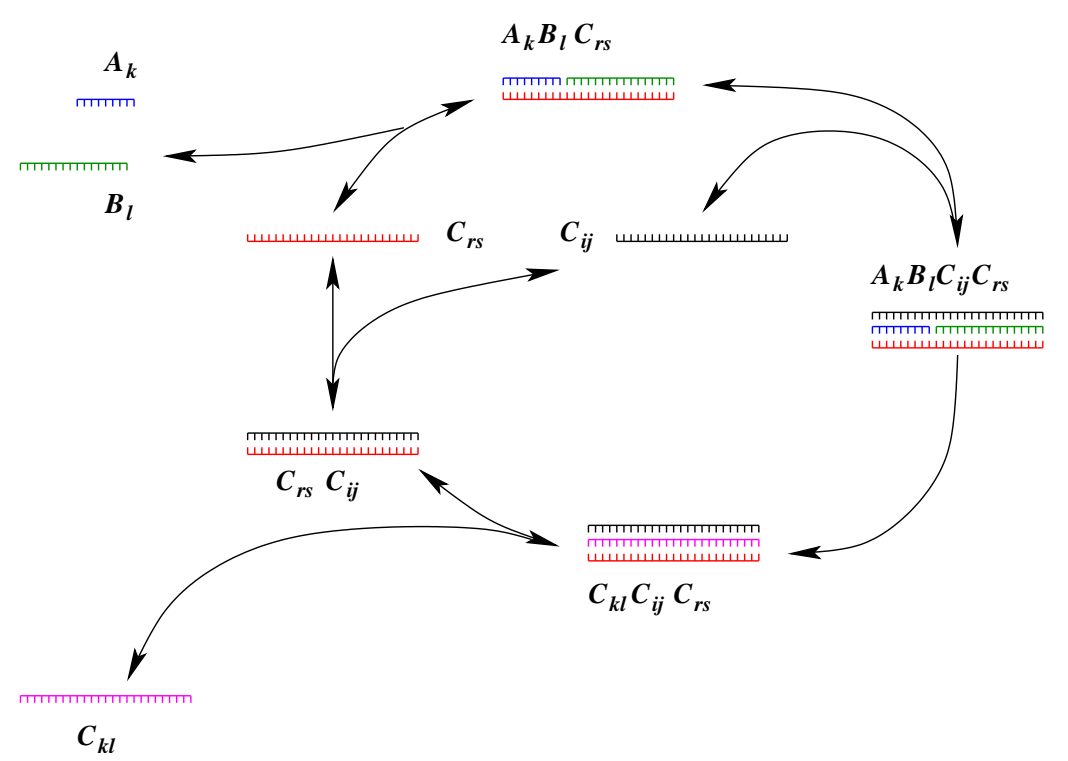

Fig. 2. A hypothetical mechanisms for actively catalyzed ligation-like replication reactions whose dynamics was studied in detail. Adapted from (Stadler et al., 2000). Note that in this scheme we have tacidly assumed that template instruction is direct rather than complementary. This amounts to assuming that all involved sequences are palindromic. Alternatively, one could complete the reaction mechanism by including a corresponding cycle for the production of offsprings from the complementary templates. It is argued e.g. in (Stadler, 1991a) that as far as the dynamics is concerned one may view a complementary pair of replicators as a single species.

Replicase Ribozymes and Higher Order Autocatalysis. Significant progress has be been made in recent years towards the construction of artificial replicase ribozymes (Ekland \& Bartel, 1996; Johnston et al., 2001; McGinness \& Joyce, 2003; Lawrence \& Bartel, 2005). While to date, no ribozyme is known that could faithfully replicate another copy of itself, this goal seems to be within experimental reach. If successful, such a riboreplicase, C, would be able of performing template directed, actively catalyzed replication, following a replication mechanism of the form:

$\mathrm{C}+\mathrm{C}+\mathrm{A} \underset{\bar{k}}{\stackrel{k}{\rightleftharpoons}} \mathrm{CC}+\mathrm{A} \underset{\bar{a}}{\stackrel{a}{\rightleftharpoons}} \mathrm{C} . \mathrm{CA} \stackrel{r}{\longrightarrow} \mathrm{C} . \mathrm{CC} \underset{\bar{d}^{\prime}}{\stackrel{d^{\prime}}{\rightleftharpoons}} \mathrm{C}+\mathrm{CC}$ and $\mathrm{CC} \underset{\bar{d}^{\prime \prime}}{\stackrel{d^{\prime \prime}}{\rightleftharpoons}} 2 \mathrm{C}$.

Theoretical models for actively catalyzed ligation-like replication are investigated in (Stadler et al., 2000). An examples are shown in Fig. 2. Molecular ecologies of strongly interacting molecular replicators have also been investigated experimentally (Wlotzka \& McCaskill, 1997; McCaskill, 1997). 


\section{Replicator Dynamics}

The mathematical analysis of the reaction schemes described in the previous section starts by translating the reaction mechanism into kinetic differential equations using the law of mass action, see e.g. (von Kiedrowski, 1993; Wills et al., 1998; Stadler et al., 2001b). As an example, consider eqn.(1). We obtain

$$
\begin{aligned}
\frac{\mathrm{d}[\mathrm{C}]}{\mathrm{d} t} & =-k[\mathrm{~A}][\mathrm{E}][\mathrm{C}]+\bar{k}[\mathrm{~A}][\mathrm{C} \cdot \mathrm{E}]+k^{\prime}[\mathrm{C} \cdot \mathrm{E} \cdot \mathrm{C}]-\bar{k}^{\prime}[\mathrm{C} \cdot \mathrm{E}][\mathrm{C}] \\
\frac{\mathrm{d}[\mathrm{C} \cdot \mathrm{E}]}{\mathrm{d} t} & =k[\mathrm{~A}][\mathrm{E}][\mathrm{C}]-\bar{k}[\mathrm{~A}][\mathrm{C} \cdot \mathrm{E}]-a[\mathrm{~A}][\mathrm{C} \cdot \mathrm{E}]+k^{\prime}[\mathrm{C} \cdot \mathrm{E} \cdot \mathrm{C}]-\bar{k}^{\prime}[\mathrm{C} \cdot \mathrm{E}][\mathrm{C}] \\
\frac{\mathrm{d}[\mathrm{C} \cdot \mathrm{E} \cdot \mathrm{C}]}{\mathrm{d} t} & =a[\mathrm{~A}][\mathrm{C} \cdot \mathrm{E}]-k^{\prime}[\mathrm{C} \cdot \mathrm{E} \cdot \mathrm{C}]+\bar{k}^{\prime}[\mathrm{C} \cdot \mathrm{E}][\mathrm{C}]
\end{aligned}
$$

Numerical integration can now be used to gain a very detailed understanding of particular model systems, provided the microscopic rate constants can be either measured directly or at least estimated. Examples include the $\mathrm{Q} \beta$ replicase system (Biebricher \& Eigen, 1988), self-replicating peptides (Islas et al., 2003), and the RNA ligase ribozyme (Bergman et al., 2000). In this contribution, however, we are interested in the qualitative and structural properties of the kinetic differential equations.

We are most interested in the total concentration $c$ of the replicator, which is the sum of free replicator concentrations $[C]$ and the concentrations of the intermediate species that contain the replicator: $c=[C]+[C E]+2[C E C]$. One observes, by adding up the differential equations for the individual contributions, that the net production of the replicator, $\dot{c}$, is determined by the single irreversible step. In the above example, this yields

$$
\dot{c}=a[\mathrm{~A}][\mathrm{C} \cdot \mathrm{E}]
$$

Under a wide variety of circumstances one can assume that the concentrations of the reaction intermediates are stationary. This is known as the quasistationary state approximation (QSSA) (Segel \& Slemrod, 1989; Borghans et al., 1996). This leads to a set of algebraic equations for the concentrations of the intermediates, which can then be substituted into the growth law for $\dot{c}$. Usually, one makes additional assumptions, e.g. that the total concentration of the enyzme $\mathrm{E}$ is constant, $\left([\mathrm{E}]+[\mathrm{C} \cdot \mathrm{E}]+[\mathrm{C} \cdot \mathrm{E} \cdot \mathrm{C}]=E_{0}\right.$ and that building material $\mathrm{A}$ is "buffered", $[\mathrm{A}]=a_{0}$ in our example.

For example, the variants of minimal replicators discussed in the previous section all lead to the same effective dynamics of the form

$$
\dot{c}=\alpha c \psi(\beta c) \quad \text { where } \quad \psi(u)=\frac{2}{u}(\sqrt{1+u}-1)
$$


where $\alpha$ and $\beta$ can be expressed in terms of the microscopic reaction rate constants. Of course, one obtains different (and usually very complicated) expressions for $\alpha$ and $\beta$ for different models. Since we will not need the explicit equations, we refer to the literature for further details (Wills et al., 1998; Stadler \& Stadler, 2003; Rasmussen et al., 2004).

The function $\psi$ appears through the solution of a quadratic equation for $[\mathrm{C}]$ in terms of $c$. Similarly, $[\mathrm{C}]$ and $c$ are related by a cubic equation in the model of enzyme-catalyzed replication, eqns.(1,7). Higher order algebraic equations also arise in the case of higher order autocatalytic systems, such as the mechanism in Fig. 2, leading to much more complex functional dependencies.

This approach readily translates to systems with different, competing, replicators $C_{k}$. In the most general case we obtain vector fields of the form

$$
\dot{c}_{k}=c_{k} F_{k}(\vec{c})
$$

where $F_{k}$ is a continuous function of the concentrations of the different replicator species. In general, it is hard or impossible to obtain a closed form for the vector field $F_{k}(\vec{c})$.

Most of the work on such coupled chemical reaction systems has been considered either a continuously stirred tank reactor (CSTR), which amounts to an additional unspecific degradation term $-r c_{k}$ or constant organization. The latter constraint fixes the total concentration $c=\sum_{k} c_{k}$ at a constant level $c_{0}$. This is equivalent to a regulated outflux $-c_{k} \Phi(\vec{c})$ which is determined by the net production of replicators:

$$
\Phi(\vec{c})=\sum_{j} \frac{c_{j}}{c} F_{j}(\vec{c}) .
$$

In the case of homogeneous interaction functions, $F_{k}(\lambda \vec{c})=h(\lambda) F_{k}(\vec{c})$, one can show that the CSTR and the constant organization model are the same up to a rescaling of the time axis (Schuster \& Sigmund, 1985). An analogous result can be shown for the limit of small flux rates $r$ in the CSTR and arbitrary interaction functions $F_{k}(\vec{c})$ (Happel \& Stadler, 1999). It is thus useful to rewrite the dynamics in terms of relative concentrations $x_{k}=c_{k} / c$. From eqn.(10) we obtain

$$
\dot{x}_{k}=x_{k}\left[F_{k}(c \cdot \vec{x})-\sum_{j} x_{j} F_{j}(c \cdot \vec{x})\right]
$$

Again, as demonstrated in (Schuster \& Sigmund, 1985), the total concentration $c$ only amounts to a re-scaling of the time axis in the case of homogeneous interaction functions $F_{k}($.$) . Eqn.(12) is the general form of a replicator equa-$ tion (Schuster \& Sigmund, 1983). This class of dynamical systems has been the 


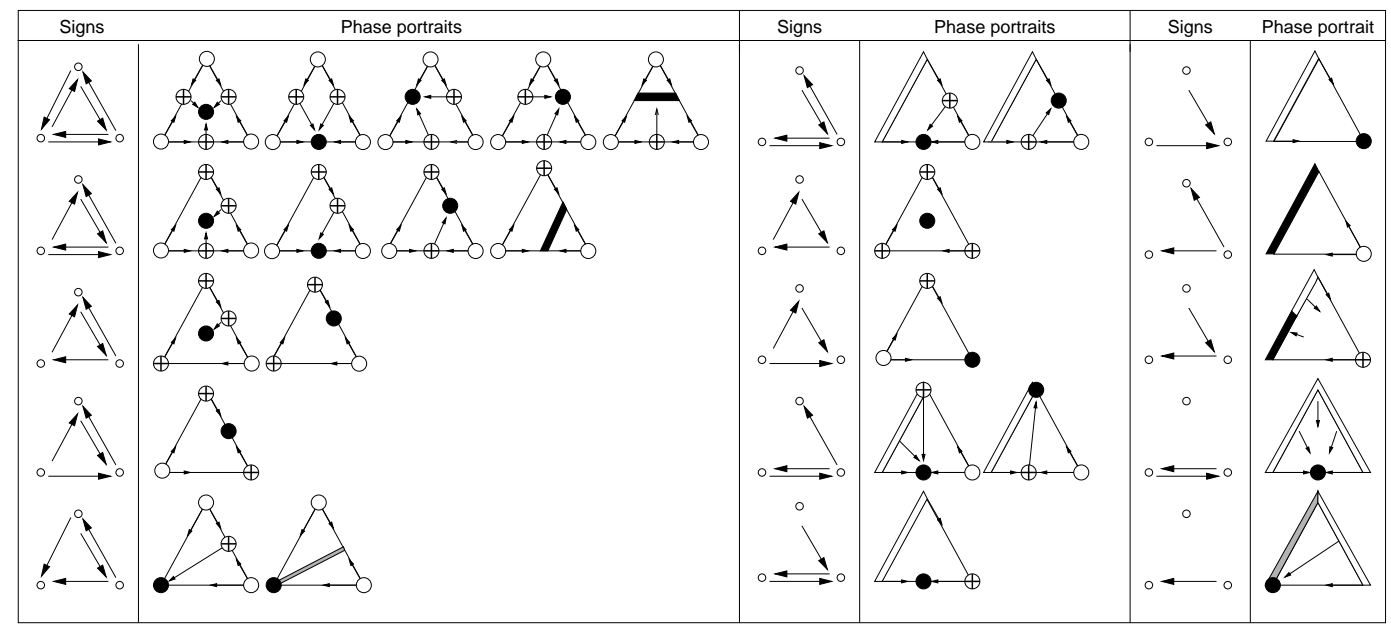

Fig. 3. Autocatalytic networks are the subclass of second order replicator equations in which there is no self-catalysis $\left(A_{i i}=0\right)$ and all other interactions are cooperative $A_{i j} \geq 0$. Their structure is readily represented by a graph with a arrow $i \rightarrow j$ iff $i$ catalyzes the replication of $j$, i.e., iff $a_{j i}>0$. The diagrams above summarize the diversity qualitative dynamical behavior of three-species autocatalytic networks. Symbols in the phase portraits: • stable fixed point (sink), o unstable fixed point (source), $\oplus$ saddle point; thick lines indicates lines consisting entirely of fixed points. Adapted from (Schuster \& Stadler, 2002).

subject of a large number of research papers as well as of the book (Hofbauer \& Sigmund, 1988).

A few cases have been studied in great detail.

- $F_{k}=a_{k}$ is a constant fitness value.

In this case we have strong selection ( "survival of the fittest"), i.e., only the sequence with the largest value of $k$ can survive.

- $F_{k}(c \cdot \vec{x})=c \sum_{j} A_{k j} x_{j}$.

These 2nd-order replicator equations also describe the dynamics of strategies in evolutionary games (Taylor \& Jonker, 1978). Hofbauer (Hofbauer, 1981) showed that they are topologically equivalent to the Lotka-Volterra equations. Their equivalence to the Price equation is demonstrated in (Page $\&$ Novak, 2002). A famous special case of a 2nd-order replicator equation is the hypercycle model of cooperative replicators (Eigen \& Schuster, 1979). Here sequence $k-1$ catalyzes the replication $k$ in a cyclic arrangement. The most important property of hypercycles is permanent coexistence, i.e., the fact that, independently of initial conditions, the relative concentrations $x_{k}$ are bounded from below by a fixed constant after a transient initial time (Schuster et al., 1979). Such cooperative behavior, however, is very rare in 2nd-order replicator equations (Happel \& Stadler, 1998; Stadler \& Happel, 1993).

The dynamics of second order replicator equations can be extremely complicated despite the rather simple form of the differential equation, Fig. 3. 

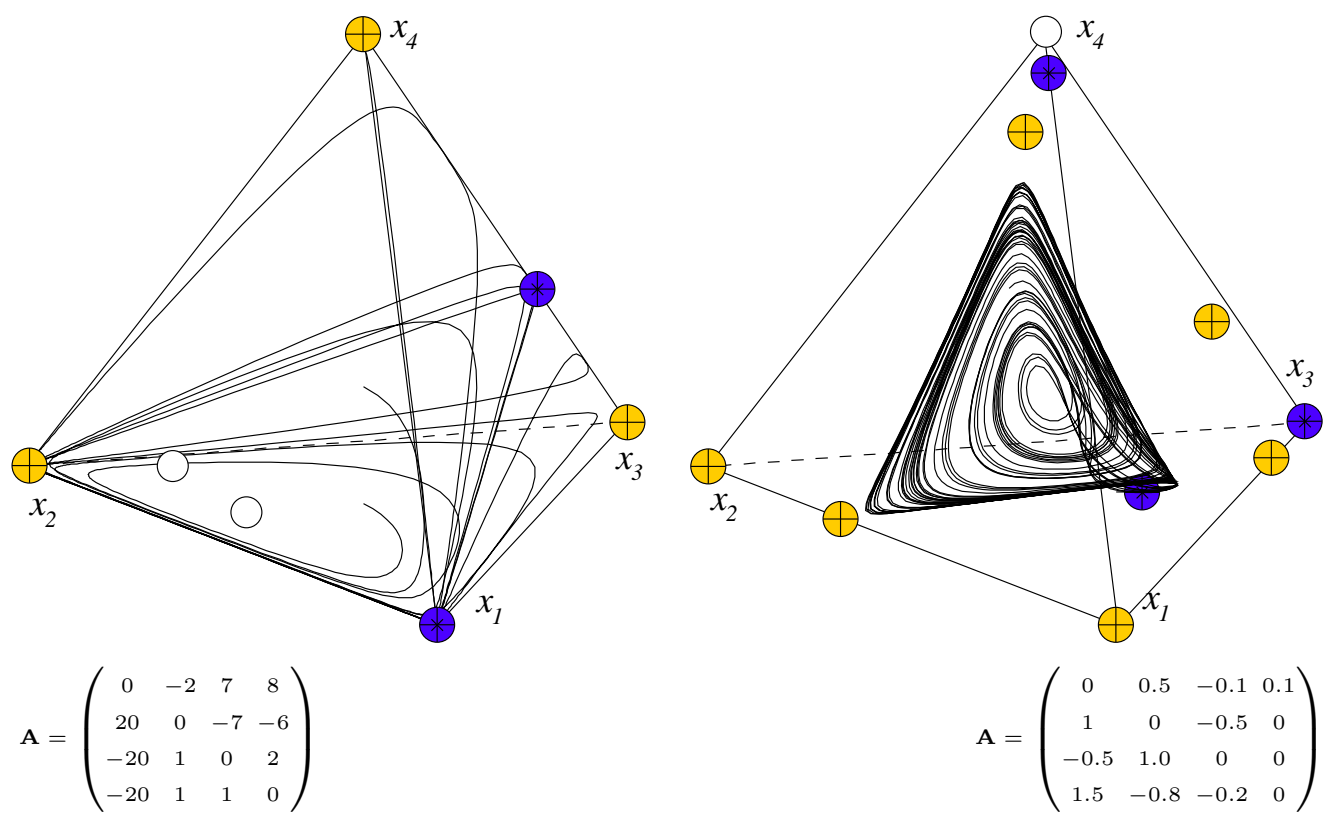

Fig. 4. Complex dynamics in 2nd order replicator equations. Left: an attracting heteroclinic orbit in the central plane (Stadler, 1996). Right: A chaotic attractor of class described in (Arneodo et al., 1980).

Fixed points are distinguished by the number of stable directions: sources without stable direction $\circ$, one stable direction $\oplus$, and $\circledast$ two stable directions.

In the case of two independent variables $(n=3$, the state space is an equilateral triangle) there are 35 different generic phase portraits (Bomze, 1983; Stadler \& Schuster, 1990). In the case of three independent variables, i.e. $n=4$ species, there are heteroclinic orbits (Brannath, 1994; Stadler, 1996), multiple limit cycles (Hofbauer \& So, 1994), and strange attractors (Gilpin, 1978; Vance, 1978; Arneodo et al., 1980; Schnabl et al., 1991; Forst, 1996), see Fig. 4

- $F_{k}(c \cdot \vec{x})=a_{k} \psi\left(c x_{k}\right)$, where $\psi$ is a monotonically decreasing function.

Such systems were investigated in detail in (Hofbauer, 1981). The minimal replicators described in the previous section are examples of this class of dynamical systems (Wills et al., 1998; Stadler et al., 2001b). There is a unique fixed point $\hat{x}$ that is eventually reached by all trajectories that start in the interior of the state space, i.e., for which all initial concentrations are non-zero. There is a survival threshold $a^{*}$ such that all species with a fitness $a_{k} \geq a^{*}$ can coexist, while those with $a_{k}<a^{*}$ eventually die out. Models with parabolic growth (von Kiedrowski, 1993; Szathmáry \& Gladkih, 1989; Varga \& Szathmáry, 1997) can be regarded as a limiting case in which the survival threshold is low enough to allow permanent coexistence (Wills et al., 1998).

- $F_{k}(c \cdot \vec{x})=\vartheta\left(c \cdot[\mathbf{A} x]_{k}\right)$, where $\vartheta$ is a monotonically increasing function. The dynamics of this system is very similar to the second order replicator equation with the same interaction matrix A (Stadler \& Stadler, 1991). 

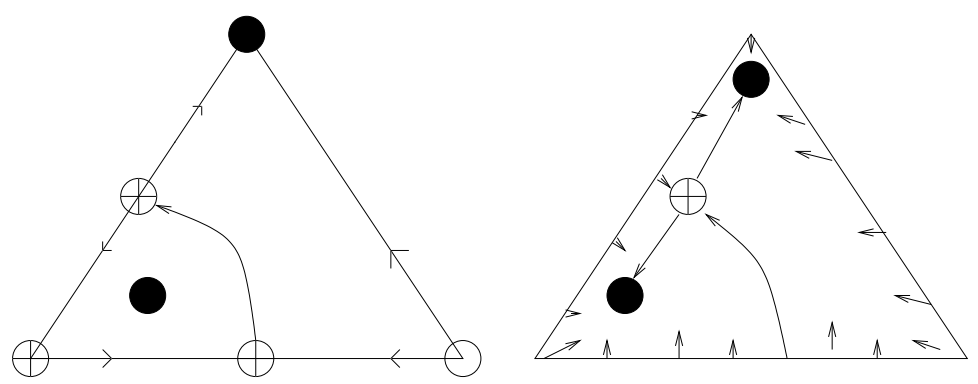

Fig. 5. Rest Point Migration Theorem.

Left: Phase portrait of selection-only system, i.e., a replicator equation. Right: The same selection part is superimposed with a mutation vector field that points inwards at the boundary. As a result, saturated fixed points are driven into the interior of the state space, while non-saturated fixed points move into the physically inaccessible exterior.

Beyond a few general results for arbitrary $F_{k}$, which are discussed in detail in (Hofbauer \& Sigmund, 1988, 1998), and the functional forms listed above, very little is known about replicator equations with non-linear response functions. A few special cases are discussed e.g. in (Hofbauer et al., 1982; Bomze, 1983; Stadler et al., 1994; Forst, 1996; Stadler et al., 2000).

\section{Replicator-Mutator Equations}

Mutation can be included in a straightforward way. Denote by $Q_{k j}$ the probability to produce an off-spring of type $k$ from a type $j$ template. The dynamics of such a system is then described by the replicator-mutator equation

$$
\dot{x}_{k}=\sum_{j} Q_{k j} x_{j} F_{j}(\vec{x})-x_{k} \Phi
$$

This expression has been used in population genetics (Hadeler, 1981), autocatalytic reaction networks (Stadler \& Schuster, 1992), game theory (Bomze \& Bürger, 1995), and language evolution (Nowak et al., 2001).

Mutation in general can be interpreted as an additional contribution to the vector field in (relative) concentration space that, at the boundary of the state space points inwards, i.e., it generates additional species that are not present in a given initial condition. Under certain conditions, namely that the offdiagonal elements in the mutation rate matrix $\mathbf{Q}$ are small enough and some (mild) technical conditions on the vector field $\vec{F}$ which are described in detail in (Stadler \& Schuster, 1992), mutation can be treated as a perturbation. Its qualitative effects on the selection dynamics are then captured by the "rest point migration theorem". 
A fixed point is saturated if it is stable against invasion, i.e., if the boundary of the concentration simplex is attracting in its vicinity. Small mutation rates deform the vector field of a replicator equation in such a way that saturated boundary equilibria move into the interior of the state space, while nonsaturated boundary equilibria move into the (non-physical) outside, Fig. 5. Small amounts of mutations therefore simplify the phase portrait of the selection dynamics and do not change stable fixed points (and limit cycles).

For constant $F_{k}$, eqn.(13) specializes to the quasispecies model (Eigen, 1971; Eigen et al., 1989). The most salient feature of this model is the existence of an error-threshold, which restricts the amount of information that can be sustained under error-prone replication. It is plausible to assume that genetic inheritance is also limited in the general case of frequency dependent selection, albeit no formal proof for this claim exists. Numerical studies for the hypercycles model are reported in (Forst, 2000).

\section{Dynamics of a Pre-Protocell}

In (Cavalier-Smith, 2001), a scenario is considered in which membranes initially functioned as supramolecular structures to which different replicators attached and were selected as a higher-level reproductive unit. This picture is conceptually simpler than micellar or vesicular protocells since it avoids the difficulties of modelling the regulation of both growth and fission. Our "preprotocell", Fig. 6, consists of a lipid aggregate that can grow by inclusion of amphiphilic molecules that are present in the environment. Attached to its surface is a suitable nucleic-acid analog, maybe some variant of a PNA, that undergoes uncatalyzed replication in the spirit of the membrane linked replication cycle of the "Los Alamos Bug" (Rasmussen et al., 2003, 2004). The type and property of a membrane fragment is determined by its inventory of genetic material.

Denote by $\Omega_{a}$ the total surface area of type- $a$ membranes and let $n_{k a}$ be the number of macromolecules with sequence $k$ embedded in it. Then

$$
\dot{n}_{k a}=n_{k a} F_{k}\left(\vec{c}_{a}\right)
$$

where $\vec{c}_{a}$ is the vector of concentrations of the different PNA sequences and $F$ is a growth law, e.g., one of those described in the previous sections. We have $c_{k a}=n_{k a} / \Omega_{a}$ and hence

$$
\dot{c}_{k a}=c_{k a} F_{k}\left(\vec{c}_{a}\right)-c_{k a} \frac{\dot{\Omega}_{a}}{\Omega_{a}}
$$

In terms of the total concentration of replicating polymers in type- $a$ mem- 


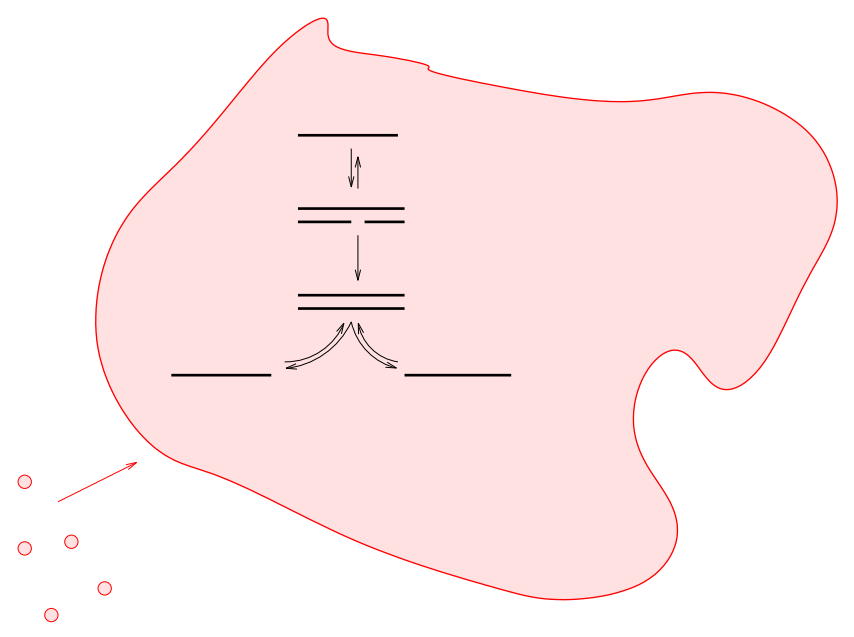

Fig. 6. Model of a Pre-protocell: Replicating polymers are attached to the surface of a lipid aggregate which can grow by incorporating amphiphilic molecules from the environment.

branes, $c_{a}=\sum_{k} c_{k a}$, and relative polymer concentrations $x_{k a}=c_{k a} / c_{a}$ (i.e., $\vec{c}_{a}=c_{a} \vec{x}_{a}$ ) we obtain an internal dynamics of the genetic material governed by the replicator equation

$$
\dot{x}_{k a}=x_{k a}\left[F_{k}\left(c_{a} \cdot \vec{x}_{a}\right)-\sum_{j} x_{j} F_{j}\left(c_{a} \cdot \vec{x}_{a}\right)\right]=x_{k a}\left[F_{k}\left(c_{a} \cdot \vec{x}_{a}\right)-\Phi_{a}\right]
$$

and a growth law for the total concentration of polymers that is linked to the membrane growth

$$
\dot{c}_{a}=c_{a} \sum_{j} x_{j} F_{j}\left(c_{a} \cdot \vec{x}_{a}\right)-c_{a} \frac{\dot{\Omega}_{a}}{\Omega_{a}}=c_{a}\left[\Phi_{a}-\frac{\dot{\Omega}_{a}}{\Omega_{a}}\right]
$$

As expected, the concentration of the genetic material is determined by the balance of replication of membrane growth. It follows directly from this equation, that a feedback is needed between the net production of genetic material $\Phi_{a}$ in the type- $a$ membrane and its growth rate $\dot{\Omega}_{a}$. If this were not the case, either the replicating material will be diluted out of the system or it will completely pack the membrane. In the latter limit, any realistic model will of course show feedback.

In order to complete this model we need to specify how the membrane growth depends on the concentrations of the attached replicators. Assuming that they have a certain catalytic activity that can increase or inhibit the incorporation of monomers into the membrane (or catalyze their formation from precursors), we expect a growth law of the general form

$$
\dot{\Omega}_{a}=\Omega_{a}\left[g+c_{a} G\left(\vec{x}, c_{a}\right)\right]
$$


We see that, as one would expect, the membrane will become asymptotically devoid of genetic material if

$$
\Phi_{a}<g
$$

i.e., if the replication rate of the polymer is smaller than the autonomous growth rate of the membrane.

On the other hand, if the polymer concentration $c_{a}$ approaches a steady state, then membrane growth is determined by the net production of replicators: $\dot{\Omega}_{a} \rightarrow \Phi_{a} \cdot \Omega_{a}$. In the case of vesicles that enclose replicating RNA, it was demonstrated that the osmotic pressure exerted on the membrane drives the uptake of membrane components from the environment (Chen et al., 2004). The growth rate of a vesicle is thus directly determined by the net production $\Phi_{a}$ of replicators in its interior.

One would expect that equations similar to those discussed above will hold for vesicular and micellar systems, with the added complication that fission of the protocells needs to be modeled. However, such cell models incur an additional complication compared to the simple membrane model discussed above: due to the small size of protocells, a certain fraction of their daughter cells will not inherit the complete set of genomic molecules and thus will not be viable. This effect further reduces the effective cellular replication rate.

In modern cells, the feedback between genomic replication and cellular growth is mediated indirectly via a complex regulatory cascade of gene expression and metabolic control. Recent advances in DNA chemistry demonstrate the possibility to "translate" the information stored in a nucleic acid sequence directly into non-polymeric compounds without the help of sophisticated enzymes (Calderone \& Liu, 2004; Gartner \& Liu, 2001; Halpin \& Harbury, 2004). Such mechanisms might provide a physico-chemical basis for the direct influence of the genome on the lipid aggregate which is implicitly postulated by the function $G\left(\vec{x}, c_{a}\right)$ above.

A particularly interesting facet of this model is the approximate dynamic independence of the individual components. In the case of homogeneous interaction functions $F_{k}$, the eventual compositions $\vec{x}_{a}$ of the genomes (mathematically speaking the $\omega$-limits of the replicator equations) are independent of the concentrations $c_{a}$. This follows immediately from the arguments in (Schuster \& Sigmund, 1985). In the limiting case of slowly varying $c_{a}$, a kind of "adiabatic" approximation allows to predict the dynamic outcomes, see (Stadler, 1991b) for some specific examples.

In this section we have presented only a cursory analysis of the dynamics of replicators interacting with a growing lipid aggregate. A more detailed investigation will be necessary to understand, for example, the conditions under which a steady-state is reached. Another research topic is to elucidate the 
relationship of the present model with simpler group-selection models that have been proposed in a prebiotic context, such as the stochastic corrector (Szathmáry \& Demeter, 1987). Clearly, the system has the potential for openended evolution. Mutations of the genetic material may lead to an increase in net flux $\Phi_{a}$, thus giving rise to a new, more competitive species. Collisions of aggregates with different genetic contents essentially play the role of "recombination".

\section{Concluding Remarks: Evolution of a Protocell Genome}

The dynamics of self-replicating hetero-polymers sets the stage for the evolution of the information that is encoded by these "proto-genomes". Indeed, a central issue in models of prebiotic evolution is the integration of information that is necessary to bridge the gap between a simple system of replicating molecules and the complexity of a modern cell (Eigen \& Schuster, 1979; Kauffman, 1993). The template length is limited by the accuracy of the replication mechanism, which is necessarily error-prone due to mutations. As an order of magnitude estimate, the length of directly replicated genome $n$ is limited by the inverse $1 / p$ of the per-digit mutation rate $p$ (Eigen, 1971). In principle the error threshold can be circumvented by evolving more accurate replicases that could be encoded by longer sequences (Scheuring et al., 2003; Poole et al., 1999; Szabó et al., 2002). Such a bootstrapping mechanism, however, requires a functional replicase-ribozyme to start with.

The error threshold, however, could be drastically relaxed in the simple model outlined above, since the dynamics of selection on this level is only weakly dependent on the dynamic details of replication. The latter could be organized cooperatively, for example in the case of a hypercycle (Eigen \& Schuster, 1979), thereby substantially increasing the genetic storage capacity. Such cooperative models are notoriously plagued by the parasite problem: Mutants without suitable catalytic activity can exploit, and eventually destroy, the entire system. Starting with the work of Boerlijst and Hogeweg (Boerlijst \& Hogeweg, 1991), it has been demonstrated, however, that the problem of parasite invasion can be alleviated by considering spatially organized systems (Streissler, 1992; Cronhjort \& Blomberg, 1994; Tereshko, 1999; Altmeyer \& McCaskill, 2001; Zintzaras et al., 2002). A replication kinetics that includes product inhibition can have a similar effect in some parameter ranges (Stadler et al., 2000, 2001b). It is conceivable that the simple coupling of replication to a container that growth under "genetic" control is already sufficient to bridge the information gap between uncatalyzed self-replication of nucleic acids with at most 20nt, and plausible replicase ribozymes, which could have a length of 100-200nt, based on a comparison with known ribozymes. 
The shape of the fitness function, and in particular the accessibility of mutants from a given population, crucially influences the dynamics of evolution (Schuster et al., 1994; Fontana \& Schuster, 1998; Stadler et al., 2001a). In the case of RNA it has been demonstrated that the sequence-structure relation is dominated by neutral mutations: single point mutations often leave structure, and thus also function, intact. This implies that functionally equivalent sequences form so-called neutral networks that percolate through sequence space. With selection acting on structure or function rather than directly on sequence, neutrality implies a significant redundancy at the sequence level and replaces the genotypic error threshold by a - relaxed- phenotypic error threshold (Forst et al., 1995; Huynen et al., 1996). It has been argued that this could be sufficient to bridge the information gap (Kun et al., 2005).

From a dynamical systems point of view, neutrality implies that the interplay of selection and mutation can efficiently explore sequence space by means of neutral drift confined to the neutral networks (Schuster et al., 1994; Huynen et al., 1996; Huynen, 1996). Recently, it was shown that a similar mechanism allows a population of autocatalytic self-replicators to explore sequence space in a diffusion-like manner (Stadler, 2002; Stephan-Otto Attolini \& Stadler, 2004).

Our simplistic pre-protocells from the previous section can therefore be expected to show all hallmarks of Darwinian evolution. They are, of course, extreme heterotrophs: we have not discussed at all where the energy-rich building material comes from that the protocells need to replicate their genomes and to grow their membranes. That, of course, is another story.

\section{Acknowledgments}

This work is supported in part by the DFG bioinformatics initiative, grant no. BIZ-6/1-2, and the COST action D27.

\section{References}

Altmeyer, S. \& McCaskill, J. S. (2001). Error threshold for spatially resolved evolution in the quasispecies model. Phys. Rev. Lett., 86:5819-5822.

Apel, C. L., Deamer, D. W., \& Mautner, M. N. (2002). Self-assembled vesicles of monocarboxylic acids and alcohols: conditions for stability and for the encapsulation of biopolymers. Biochim. Biophys. Acta, 1559:1-9.

Arneodo, A., Coullet, P., \& Tresser, C. (1980). Occurence of strange attractors in three-dimensional Volterra equations. Phys. Lett. A, 79:259-263. 
Ashkenazy, G., Jagasia, R., Yadav, M., \& Ghadiri, M. R. (2004). Design of a directed molecular network. Proc. Natl. Acad. Sci. USA, 101:10872-10877.

Bergman, N. H., Johnston, W. K., \& Bartel, D. P. (2000). Kinetic framework for ligation by an efficient RNA ligase ribozyme. Biochemistry, 39:31153123.

Biebricher, C. K. \& Eigen, M. (1988). Kinetics of RNA replication by Q $\beta$ replicase. In E. Domingo, J. J. Holland, \& P. Ahlquist (Eds.), RNA Genetics. Vol.I: RNA Directed Virus Replication, (pp. 1-21). Boca Raton, FL: CRC Press.

Biebricher, C. K., Eigen, M., \& Gardiner jr, W. C. (1983). Kinetics of rna replication. Biochemistry, 22:2544-2559.

Boerlijst, M. C. \& Hogeweg, P. (1991). Spiral wave structure in pre-biotic evolution: hypercycles stable against parasites. Physica D, 48:17-28.

Bomze, I. (1983). Lotka-Volterra equations and replicator dynamics: A twodimensional classification. Biol. Cybern., 48:201-211.

Bomze, I. \& Bürger, R. (1995). Stability by mutation in evolutionary games. Games Econ. Behav., 11:146-172.

Borghans, J. A. M., de Boer, R. J., \& Segel, L. A. (1996). Extending the quasi-steady state approximation by changing variables. Bull. Math. Biol., 58:43-63.

Brannath, W. (1994). Heteroclinic networks on the tetrahedron. Nonlinearity, 7:1367-1384.

Calderone, C. T. \& Liu, D. R. (2004). Nucleic-acid-templated synthesis as a model system for ancient translation. Curr. Op. Chem. Biol., 8:645-653.

Cavalier-Smith, T. (2001). Obcells as proto-organisms: membrane heredity, lithophosphorylation, and the origins of the genetic code, the first cells, and photosynthesis. J. Mol. Evol., 53:555-595.

Chen, I. A., Roberts, R. W., \& Szostak, J. W. (2004). The emergence of competition between model protocells. Science, 305:1474-1476.

Cronhjort, M. B. \& Blomberg, C. (1994). Hypercycles versus parasites in a two dimensional partial differential equations model. J. Theor. Biol., 169:31-49.

Dawkins, R. (1976). The Selfish Gene. Oxford: Oxford University Press.

Eigen, M. (1971). Selforganization of matter and the evolution of biological macromolecules. Naturwissenschaften, 58:465-523.

Eigen, M., McCaskill, J. S., \& Schuster, P. (1989). The molecular quasi-species. Adv. Chem. Phys., 75:149-263.

Eigen, M. \& Schuster, P. (1979). The Hypercycle. New York, Berlin: SpringerVerlag.

Ekland, E. H. \& Bartel, D. P. (1996). RNA-catalysed RNA polymerization using nucleoside triphosphates. Nature, 382:373-376.

Fontana, W. \& Schuster, P. (1998). Continuity in evolution: On the nature of transitions. Science, 280:1451-1455.

Forst, C. V. (1996). Chaotic interactions of self-replicating rna. Computers \&6 Chem., 20:69-83.

Forst, C. V. (2000). Molecular evolution of catalysis. J. Theor. Biol., 205:409- 
431.

Forst, C. V., Reidys, C. M., \& Weber, J. (1995). Evolutionary dynamics and optimization: Neutral Networks as model-landscape for RNA secondarystructure folding-landscapes. In F. Morán, A. Moreno, J. Merelo, \& P. Chacón (Eds.), Advances in Artificial Life, vol. 929 of Lecture Notes in Artificial Intelligence, (pp. 128-147). ECAL '95, Berlin, Heidelberg, New York: Springer.

Gartner, Z. J. \& Liu, D. R. (2001). The generality of DNA-templated synthesis as a basis for evolving nonnatural small molecules. J. Amer. Chem. Soc., 123:6961-6963.

Gilpin, M. E. (1978). Spiral chaos in a predator prey system. Amer. Nat., 133:306-308.

Hadeler, K. P. (1981). Stable polymorphisms in a selection model with mutation. SIAM J. Appl. Math., 41:1-7.

Halpin, D. R. \& Harbury, P. B. (2004). DNA display i. sequence-encoded routing of DNA populations. PLoS Biol, 2:e173. DOI: 10.1371/journal.pbio.0020173.

Happel, R. \& Stadler, P. F. (1998). The evolution of diversity in replicator networks. J. Theor. Biol., 195:329-338.

Happel, R. \& Stadler, P. F. (1999). Autocatalytic replication in a CSTR and constant organization. J. Math. Biol., 38:422-434.

Hofbauer, J. (1981). On the occurrence of limit cycles in Volterra-Lotka equations. Nonlin. Anal., 5:1003-1007.

Hofbauer, J., Schuster, P., \& Sigmund, K. (1982). Game dynamics in mendelian populations. Biol. Cybern, 43:51-57.

Hofbauer, J. \& Sigmund, K. (1988). Dynamical Systems and the Theory of Evolution. Cambridge U.K.: Cambridge University Press.

Hofbauer, J. \& Sigmund, K. (1998). Evolutionary Games and Population Dynamics. Cambridge U.K.: Cambridge University Press.

Hofbauer, J. \& So, J. (1994). Multiple limit cycles for three-dimensional LotkaVolterra equations. Appl. Math. Lett., 7:65-70.

Huynen, M. A. (1996). Exploring phenotype space through neutral evolution. J. Mol. Evol., 43:165-169.

Huynen, M. A., Stadler, P. F., \& Fontana, W. (1996). Smoothness within ruggedness: the role of neutrality in adaptation. Proc. Natl. Acad. Sci. (USA), 93:397-401. SFI preprint 95-01-006, LAUR-94-3763.

Isaac, R. \& Chmieleswski, J. (2002). Approaching exponential growth with a self-replicating peptide. J. Amer. Chem. Soc., 124:6808-6809.

Islas, J. M., Pimienta, V., Micheau, J.-C., \& Buhse, T. (2003). Kinetic analysis of artificial peptide self-replication: Part I: the homochiral case. Biophys. Chem., 103:191-200.

Johnston, W. K., Unrau, P. J., Lawrence, M. J., Glasner, M. E., \& Bartel, D. P. (2001). RNA-catalyzed RNA polymerization: Accurate and general RNA-templated primer extension. Science, 292:1319-1325.

Joyce, G. F. (2002). The antiquity of RNA-based evolution. Nature, 418:214- 
221.

Kauffman, S. A. (1993). The Origin of Order. New York, Oxford: Oxford University Press.

Kun, A., Santos, M., \& Szathmáry, E. (2005). Real ribozymes suggest a relaxed error threshold. Nature Genetics, 37:1008-1011.

Lawrence, M. S. \& Bartel, D. P. (2005). New ligase-derived RNA polymerase ribozymes. $R N A, 11: 1173-1180$.

Lee, D. H., Granja, J. R., Martinez, J. A., Severin, K., \& Ghadiri, M. R. (1996). A self-replicating peptide. Nature, 382:525-528.

Lee, D. H., Severin, K., \& Ghadiri, M. R. (1997). Autocatalytic networks: the transition from molecular self-replication to ecosystems. Curr. Opin. Chem. Biol., 1:491-496.

Li, T. \& Nicolaou, K. C. (1994). Chemical self-replication of palindromic duplex DNA. Nature, 369:218-221.

Luisi, P. L., Walde, P., \& Oberholzer, T. (1994). Enzymatic RNA synthesis in self-reproducing vesicles: An approach to the construction of a minimal synthetic cell. Ber. Bunsenges. Phys. Chem., 98:1160-1165.

McCaskill, J. S. (1997). Spatially resolved in vitro molecular ecology. Biophys Chem, 66:145-158.

McGinness, K. E. \& Joyce, G. F. (2003). In search of an RNA replicase ribozyme. Chem. Biol., 10:5-14.

Mills, D. R., Peterson, R. L., \& Spiegelman, S. (1967). An extracellular Darwinian experiment with a self-duplicating nucleic acid molecule. Proc. Natl. Acad. Sci. USA, 58:217-224.

Nielsen, P. E. (1993). Peptide nucleic acid (PNA): a model structure for the primordial genetic material? Orig. Life Evol. Biosph., 23:323-327.

Nowak, M. A., Komarova, N. L., \& Niyogi, P. (2001). Evolution of universal grammar. Science, 291:114-118.

Page, K. M. \& Novak, M. A. (2002). Unifying evolutionary dynamics. J. Theor. Biol., 219:93-98.

Paul, N. \& Joyce, G. F. (2003). A self-replicating ligase ribozyme. Proc. Natl. Acad. Sci. USA, 99:12733-12740.

Paul, N. \& Joyce, G. F. (2004). Minimal self-replicating systems. Curr. Op. Chem. Biol., 8:634-639.

Pohorille, A. \& Deamer, D. (2002). Artificial cells: prospects for biotechnology. Trends Biotech., 20:123-128.

Poole, A., Jeffares, D., \& Penny, D. (1999). Early evolution: prokaryotes, the new kids on the block. Bioessays, 21:880-889.

Rasmussen, S., Chen, L., Nilsson, M., \& Abe, S. (2003). Bridging nonliving and living matter. Artificial Life, 9:269-316.

Rasmussen, S., Chen, L., Stadler, B. M. R., \& Stadler, P. F. (2004). Protoorganism kinetics: Evolutionary dynamics of lipid aggregates with genes and metabolism. Orig. Life Evol. Biosph., 34:171-180.

Scheuring, I., Czaran, T., Szabo, P., Karyoli, G., \& Toroczkai, Z. (2003). Spatial models of prebiotic evolution: Soup before pizza? Origins of life, 33(4- 
5):329-355.

Schnabl, W., Stadler, P. F., Forst, C., \& Schuster, P. (1991). Full characterization of a strange attractor. Physica D, 48:65-90.

Schuster, P., Fontana, W., Stadler, P. F., \& Hofacker, I. L. (1994). From sequences to shapes and back: A case study in RNA secondary structures. Proc. Roy. Soc. Lond., B225:279-284.

Schuster, P. \& Sigmund, K. (1983). Replicator dynamics. J. Theor. Biol., 100:533-538.

Schuster, P. \& Sigmund, K. (1985). Dynamics of evolutionary optimization. Ber. Bunsenges. Phys. Chem., 89:668-682.

Schuster, P., Sigmund, K., \& Wolff, R. (1979). Dynamical systems under constant organization III: Cooperative and competitive behaviour of hypercycles. J. Diff. Eqns., 32:357-368.

Schuster, P. \& Stadler, P. F. (2002). Networks in molecular evolutions. Complexity, 8:34-42.

Segel, L. A. \& Slemrod, M. (1989). The quasi-steady state assumption: a case study in perturbation. SIAM Rev., 31:446-477.

Spiegelman, S. (1971). An approach to the experimental analysis of precellular evolution. Quart. Rev. Biophys., 4:213-253.

Stadler, B. M. R. (1996). Segregation distortion and heteroclinic cycles. J. Theor. Biol., 183:363-379.

Stadler, B. M. R. (2002). Diffusion of a population of interacting replicators in sequence space. Adv. Complex Systems, 5(4):457-461.

Stadler, B. M. R. \& Stadler, P. F. (1991). Dynamics of small autocatalytic reaction networks III: Monotonous growth functions. Bull. Math. Biol., 53:469-485.

Stadler, B. M. R. \& Stadler, P. F. (2003). Molcular replicator dynamics. Adv. Complex Syst., 6:47-77. Presented at Emergence in Chemical Systems, Anchorage AK, June 2002; SFI \# 02-09-049.

Stadler, B. M. R., Stadler, P. F., \& Schuster, P. (2000). Dynamics of autocatalytic replicator networks based on higher order ligation reactions. Bull. Math. Biol., 62:1061-1086.

Stadler, B. M. R., Stadler, P. F., Wagner, G., \& Fontana, W. (2001a). The topology of the possible: Formal spaces underlying patterns of evolutionary change. J. Theor. Biol., 213:241-274.

Stadler, B. M. R., Stadler, P. F., \& Wills, P. R. (2001b). Evolution in systems of ligation-based replicators. Z. Phys. Chem., 21-33:216.

Stadler, P. F. (1991a). Complementary replication. Math. Biosc., 107:83-109.

Stadler, P. F. (1991b). Dynamics of small autocatalytic reaction network IV: Inhomogeneous replicator equations. BioSystems, 26:1-19.

Stadler, P. F. \& Happel, R. (1993). The probability for permanence. Math. Biosc., 113:25-50.

Stadler, P. F. \& Schuster, P. (1990). Dynamics of small autocatalytic reaction networks I: Bifurcations, permanence and exclusion. Bull. Math. Biol., 52:485-508. 
Stadler, P. F. \& Schuster, P. (1992). Mutation in autocatalytic networks an analysis based on perturbation theory. J. Math. Biol., 30:597-631.

Stadler, P. F., Schuster, P., \& Perelson, A. S. (1994). Immune networks modelled by replicator equations. J. Math. Biol., 33:111-137.

Stephan-Otto Attolini, C. \& Stadler, P. F. (2004). Evolving towards the hypercycle: A spatial model of molecular evolution. Physica D. Submitted.

Streissler, C. (1992). Autocatalytic Networks Under Diffusion. Ph.D. thesis, University of Vienna.

Szabó, P., Scheuring, I., Czaran, T., \& Szathmáry, E. (2002). In silico simulations reveal that replicators with limited dispersal evolve towards higher efficiency and fidelity. Nature, 420:278-279.

Szathmáry, E. \& Demeter, L. (1987). Group selection of early replicators and the orgin of life. J. Theor. Biol., 128:463-486.

Szathmáry, E. \& Gladkih, I. (1989). Sub-exponential growth and coexistence of non-enzymatically replicating templates. J. Theor. Biol., 138:55-58.

Szostak, J., Bartel, D., \& Luisi, P. L. (2001). Synthesizing life. Nature, 409 Suppl:387-390.

Taylor, P. D. \& Jonker, L. B. (1978). Evolutionary stable strategies and game dynamics. Math.Biosc., 40:145-156.

Tereshko, V. (1999). Selection and coexistence by reaction-diffusion dynamics in fitness landscapes. Phys. Let. A, 260:522-527.

Tijvikua, T., Ballester, P., \& Rebek jr, J. (1990). A self-replicating system. J. Amer. Chem. Soc., 112:1249-1250.

Vance, R. R. (1978). Predation and resource partitioning in one predator two prey model communities. Amer. Nat., 112:797-813.

Varga, S. \& Szathmáry, E. (1997). An extremum principle for parabolic competition. Bull. Math. Biol., 59:1145-1154.

von Kiedrowski, G. (1986). A self-replicating hexadeoxynucleotide. Angew. Chem. Int. Ed. Engl., 25:932-935.

von Kiedrowski, G. (1993). Minimal replicator theory I: Parabolic versus exponential growth. In Bioorganic Chemistry Frontiers, Volume 3, (pp. 115-146). Berlin, Heidelberg: Springer-Verlag.

Vrba, E. S. (1989). Levels of selection and sorting with special reference to the species level. In P. H. Harvey \& L. Partridge (Eds.), Oxford Surveys in Evolutionary Biology, vol. 6, (pp. 114-115). Oxford: Oxford University Press.

Whitten, D. G., Chen, L., Geiger, H. C., Perlstein, J., \& Song, X. (1998). Selfassembly of aromatic-functionalized amphiphiles: The role and consequences of aromatic-aromatic noncovalent interactions in building supramolecular aggregates and novel assemblies. J. Phys. Chem. B, 102:10098-10111.

Wills, P. R., Kauffman, S. A., Stadler, B. M., \& Stadler, P. F. (1998). Selection dynamics in autocatalytic systems: Templates replicating through binary ligation. Bull. Math. Biol., 60:1073-1098.

Wintner, E. A., Conn, M. M., \& Rebek jr, J. (1994). Self-replicating molecules: a second generation. J. Amer. Chem. Soc., 116:8877-8884. 
Wlotzka, B. \& McCaskill, J. S. (1997). A molecular predator and its prey: Coupled isothermal amplification of nucleic acids. Chemistry $\&$ Biology, $4: 25-33$.

Yao, S., Ghosh, I., Zutshi, R., \& Chmielewski, J. (1998). Selective amplification by auto- and cross-catalysis in a replicating peptide system. Nature, 396:447-450.

Zielinski, W. S. \& Orgel, L. E. (1987). Autocatalytic synthesis of a tetranucleotide analogue. Nature, 327:346-347.

Zintzaras, E., Santos, M., \& Szathmáry, E. (2002). "Living" under the challenge of information decay: The stochastic corrector model vs. hypercycles. J. Theor. Biol., 217:167-181. 